\title{
Comparison between Algorithms of Deep Learning to Detect Brain Tumor
}

\author{
Jinal Jain ${ }^{a}$, Dr.Rajesh Bansode ${ }^{b}$ \\ Thakur College of Engineering and Technology, Kandivali, India ${ }^{\mathrm{a}, \mathrm{b}}$ \\ jinaljain12@gmail.com ${ }^{\mathrm{a}}$, rajesh.bansode@thakureducation.org ${ }^{\mathrm{b}}$
}

\begin{abstract}
Deep Learning has grown considerably in recent years and has sparked a lot of interest in determining brain size and technical characteristics in brain tumour identification over the last few years. The healthcare industry is one of the domains of business where there is a lot of risk, and it has attracted the attention of a lot of technological companies, so it requires a lot of precision to get good results. There are several potential for the healthcare industry to achieve and benefit by utilising deep learning algorithms. The algorithms' new offering is a more comprehensive, revolutionary package of products and expert services that address a broader range of essential business issues. Deep learning algorithms may give significant benefits in the context of smart healthcare systems, particularly from a situation perspective where efficient solutions can be delivered to citizens and society. The primary goal of this proposed methodology is to deliver simple, quick, and accurate findings in the identification of brain tumours. CNN, VGG16, VGG19, and Resnet50 are four deep learning algorithms implemented in this system. The results of all four algorithms are compared, and a judgement is formed as to which method is best suited for the most accurate detection of brain tumor. The VGG16 algorithm, which is the most accurate and trustworthy of the four algorithms, achieves an accuracy of 89 percent.
\end{abstract}

Keywords: DTI, MRI, MRS, PET, Precision, F1-score, Recall, Cohen's Kappa, VGG16, VGG19, ResNet50, CNN.

\section{Introduction}

Cancer is characterised as the unregulated and abnormal division and growth of cells in the body. The occurrence of these unnatural cell proliferation and differentiation in the brain tissue as a mass is referred to as a brain tumour. Brain tumours are one of the most deadly cancers, despite their rarity. Any growth inside skull, restricted space, can cause problems. When viewed through a microscope, they stay small and have a normal appearance. Tumor is categorized based on a molecular signature in tumor cell with distinguishing characteristics. Some cells lose their competency to regulate their growth-they grow deprived of any order. Tumor which originates in brain is called as Primary tumors. For this form of tumour grade, surgery may be a viable option. Tumor is categorized based on a molecular signature in tumor cell with distinguishing characteristics. Tumors can be malignant (cancerous) or benign (noncancerous) 1-2. Some cells lose their competency to regulate their growth-they grow deprived of any order which is known as tumor. Also, in India, there is steep rise of brain tumor cases which are reported accurately. Among all age groups, cases of brain tumor are reported each year in India which is a topic of concern for overall growth of nation.

Risk factors play important role in acquiring brain tumor. Under Family history, minimum of about 5 to $10 \%$ of all type of cancers are hereditary. With age, the risk of brain tumor increases. Another 
important factor of brain tumor is the fact that in work environment people are exposed to certain chemicals which can increase risk of brain tumor. Similarly, people who are in contact with ionizing radiation have an increased risk of brain tumors. According to ABTA, if once detected with childhood chickenpox there is a decreased risk of acquiring brain tumor 3. Below is the statics of rising brain tumor of past few years as shown below in fig. $1-$

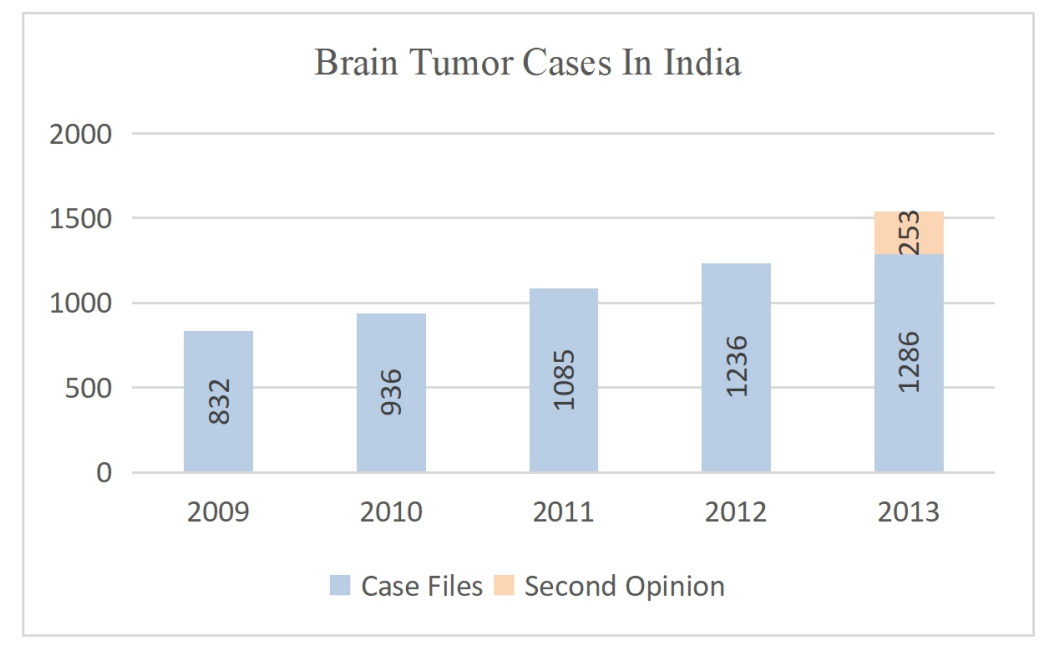

Figure 01: Statistics of increasing brain tumor cases in India

Deep learning approaches are effective for segmentation, with the Convolutional Neural Network $(\mathrm{CNN})$ often used in particular for pattern recognition 4-5. This architecture replaces the current mechanism which is a cumbersome process of brain tumor detection performed manually which takes a lot of time. Here, an automated system is constructed that will eliminate all time-consuming processes and provide results in no time.

Brain tumor detection is divided into two parts. First brain denoted as A is brain with no tumor and the second brain is with tumor as below in fig 2 -
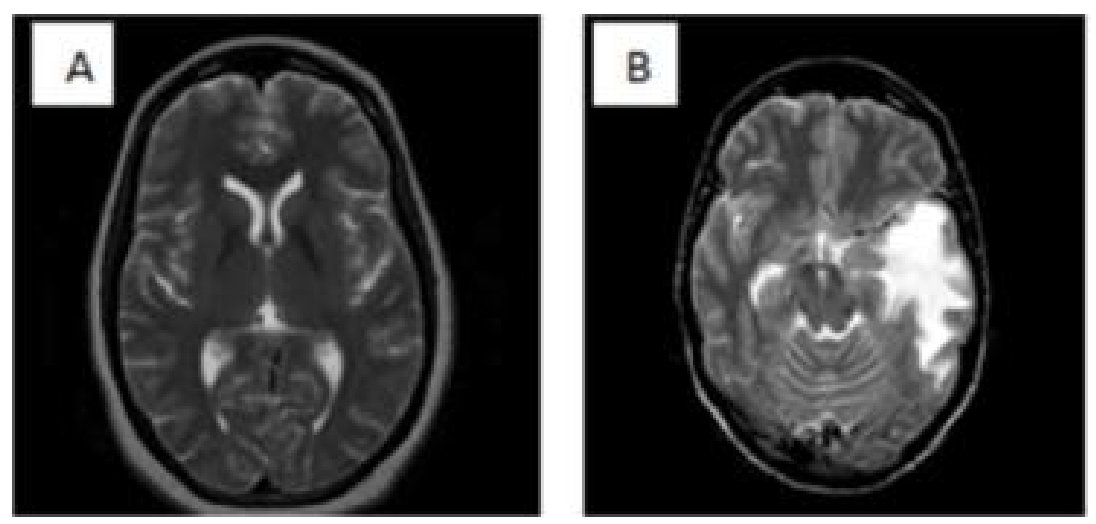

Figure 02: A) Brain without tumor (Normal brain) and B) Brain with tumor

Here, classification is made between normal brain and brain with tumor. So basically, 5 classes are created viz., class 0 - DTI scanned brain tumor images, Class 1 - MRI scanned brain tumor images, Class 2 - MRS scanned brain tumor images, Class 3 - Normal brain scan images which does not have tumor, Class 4 - PET scanned brain tumor images as in fig 2. Normal brain scanned images without 
International Journal of Intelligent Communication, Computing and Networks

Open Access Journal (ISSN: 2582-7707)

https://doi.org/10.51735/ijiccn/001/23

tumor includes of all different scans. In this paper, 3-4 algorithms will be applied. Parameters that will be used are accuracy, recall, f1-score, Cohen's kappa, error loss and precision.

\subsection{Literature Review}

Tanzila Saba et al., (2020) conducted a thorough investigation into Healthcare Industry regarding brain tumor detection. The Grab cut approach was discussed for image positioning of actual lesion symptoms, while the Transfer learning model visual geometry community (VGG-19) was fine-tuned to acquire features that were then concatenated with hand-crafted (shape and texture) features using a serial-based method. However, usage of multiple brain scans is neglected. Current technology's limitations must be identified, and future research guidelines must be considered 1 .

Ambeshwar Kumar et al., (2020) used the region based semantic segmentation and SVM classifier. Also CNN and MLP algorithms are applied on the images. Current e-Healthcare systems, on the other hand, still aren't properly matured and developed, and therefore lack the degree of correctness needed. The diagnosis process is being difficult to be performed in the final stages of disease 2 .

Sidra Sajid et al., (2019) propose a deep learning architecture with MRI brain scans and CNN algorithm. Two path CNN and Three Path CNN algorithms are used. Accuracy achieved here need to be revised as per the ever changing and demanding healthcare industry 3 .

Abd El Kader Isselmou et al., (2020) proposed the detection of brain tumour using MRI brain scanned images by using CNN algorithm. The proposed structure has been extensively tested using MRI images of patients with different types of brain tumors as a data base. CSS concepts are used in order to achieve high accuracy. Using sensitivity, specificity, recall, and precision values, the study findings presented the output of the suggested technique for brain tumor detection and analysis based on deep learning network 4.

Heba Mohsen et al., (2018) for labelling the database, a Deep Neural Network classifier has been used, that's one of the DL architectures. The classification model was associated with the efficient feature extraction tool discrete wavelet transform (DWT) and principal component analysis (PCA). This data must be carefully managed and analysed in order to obtain valuable information. In the possibility, the positive results obtained with the DWT may be used with the CNN to analyse the outcomes 5 .

Javeria Amin et al., (2017) proposed to distinguish among cancerous and non-cancerous brain Magnetic Resonance Imaging (MRI). For the segmentation of candidate lesions, various techniques have been used. Then, based on form, texture, and severity, a feature set for each applicant lesion is selected. The precision of the proposed architecture is then compared using a Support Vector Machine (SVM) classifier with different cross verification on the features collection. How to process images with more accuracy in less time is also discussed. The proposed technique can be used to improve tumor detection procedures prior to the onset of complications 6 .

AliIşın et al., (2016) centred on the field's recent trend of deep learning methods. First, a brief overview of brain tumours and strategies for segmenting them is given. The state-of-the-art algorithms are then addressed, with an emphasis on the current developments of deep learning methods. Finally, 
an evaluation of the existing state is provided, as well as potential advances aimed at integrating MRI-based brain tumor segmentation approaches into everyday clinical practice 7 .

D,N, Louis et al., (2016) new neoplasms have been introduced, and some entities, mutations, and behaviors and were no further functional or genetically appropriate have been removed. As a consequence, molecular markers are now used to recognize brain tumors. However, the research lacks implementation of other brain scans which will cover wider range in order to get to results as per growing healthcare industry 8 .

Abdel Maksoud Eman et al., (2015) present a quick image segmentation method that combines the K-means clustering technique with the Fuzzy C-means algorithm. In terms of accuracy, processing time, and efficiency, the proposed image segmentation method was compared to some state-of-the-art segmentation algorithms. Author illustrates basic issues and benefits of hybrid clustering techniques for deploying a reliable and scalable solution for brain tumor detection in order to achieve the best possible output 9 .

Tuhin Utsab Paul et al., (2012) present detection of brain tumor by using MRI images. The proposed method for segmentation solves the limitations of the traditional K-means algorithm and produces very good result from both a qualitative and quantitative standpoint. Furthermore, the segmentation prediction accuracy in brain MRI images collected from all three perspectives is very high and appropriate 10 .

\subsection{Problem Statement}

Deep learning is a viable technology that can enhance the healthcare detection of brain tumor. Low accuracy and high error rate is one of the most serious problems in current technology. Data gathering, data analysis and data preprocessing is the main concern on which the entire system relies. Model selection too plays a very important role in deep learning. Bad model if selected will always give low accuracy and hgh error rate which is the main concern here.

\subsection{Use of Deep Learning in Healthcare}

Several deep learning algorithms in the healthcare sector have shown that the platform can be beneficial in a number of ways. There are various other algorithms too available like K-means or SVM that prove to be useful and which gives promising results 6. With Deep Learning we get high accuracy and low error rate as the dataset is huge and algorithms are complex and reliable. Fuzzy logic also is used to be useful which could be relied upon for getting the best results. Furthermore, dataset is also been taken from reliable sources which could be Kaggle or UCI or BRATS 7. In this scenario. Also, Healthcare industry is daily rising and upcoming with solutions to various diseases so we need to be updated.

\subsection{Motivation}


1) In 2018 , in India, amongst the common kind of tumors, brain tumor was ranked as the $10^{\text {th }}$ position.

2) Over 28,000 cases of brain tumors reported each year, and more than 24,000 people reportedly die in India as reported by The International Association of Cancer Registries (IACR) 8.

3) A brain tumor is a serious condition and can be fatal if not detected early and treated

4) Tumor which are malignant ones are cancerous, they are growing disorderly and have ability to spread to other parts of the body.

\subsection{Objective}

1) The goal of our study is to use deep learning to create an automated image-based system for detecting brain tumour illness.

2) To arrive at a conclusion, deep learning algorithms use numerous layers and the softmax function.

3) We will use a variety of algorithms in this system to help improve accuracy

4) Several algorithms are employed to find the best algorithm with the maximum accuracy. The system will classify tumor between normal (benign) and malign (glioblastoma, sarcoma and metastatic bronchogenic carcinoma tumors) 9.

5) Algorithms will be compared based on their accuracy, error rate, precision, recall, f1-score, and Cohen's Kappa results.

\subsection{Issues and Challenges}

Most conventional artificial intelligence systems are "black boxes," making it difficult to validate, change, or refine the study 10. Leaders are left to question if these systems are successful or how they can be improved. Educating data science concepts and skills to existing squad is a time-consuming, challenging, and unrealistic mission. The vast majority of AI tools are standalone systems that necessitate analysts learning and applying yet another piece of software, language, or connection process.

\subsection{Proposed System}

The proposed system's primary goal is to analyse and determine whether or not an image contains a brain tumor. In this system we will be taking images of brain scan of not only MRI but also of MRS, PET and DTI brain scans in order to get a fuller coverage of brain tumor detection. On these photos, we'll use a variety of deep learning algorithms to determine whether the image of a brain scan contains a tumour at any particular place. Image gathering is done via open source Kaggle dataset and via web scrapping. Later images gathered by web scrapping is cleaned and various techniques are applied to 
make it algorithm ready. Duplicate images are removed and then data augmentation is applied on the images. By data augmentation, images are rotated, flipped, zoomed and other such techniques are applied so that we get more no of images. This is done because in any kind of deep learning algorithms, large dataset is the key. This dataset is then applied on the algorithms to get the best algorithm after comparing the output parameters. Then the system is ready and is deployed. So later if any image is given to the system then the system will be able to recognize if the scanned image of brain contains a tumor.

\subsection{Proposed Methdology Architecture}

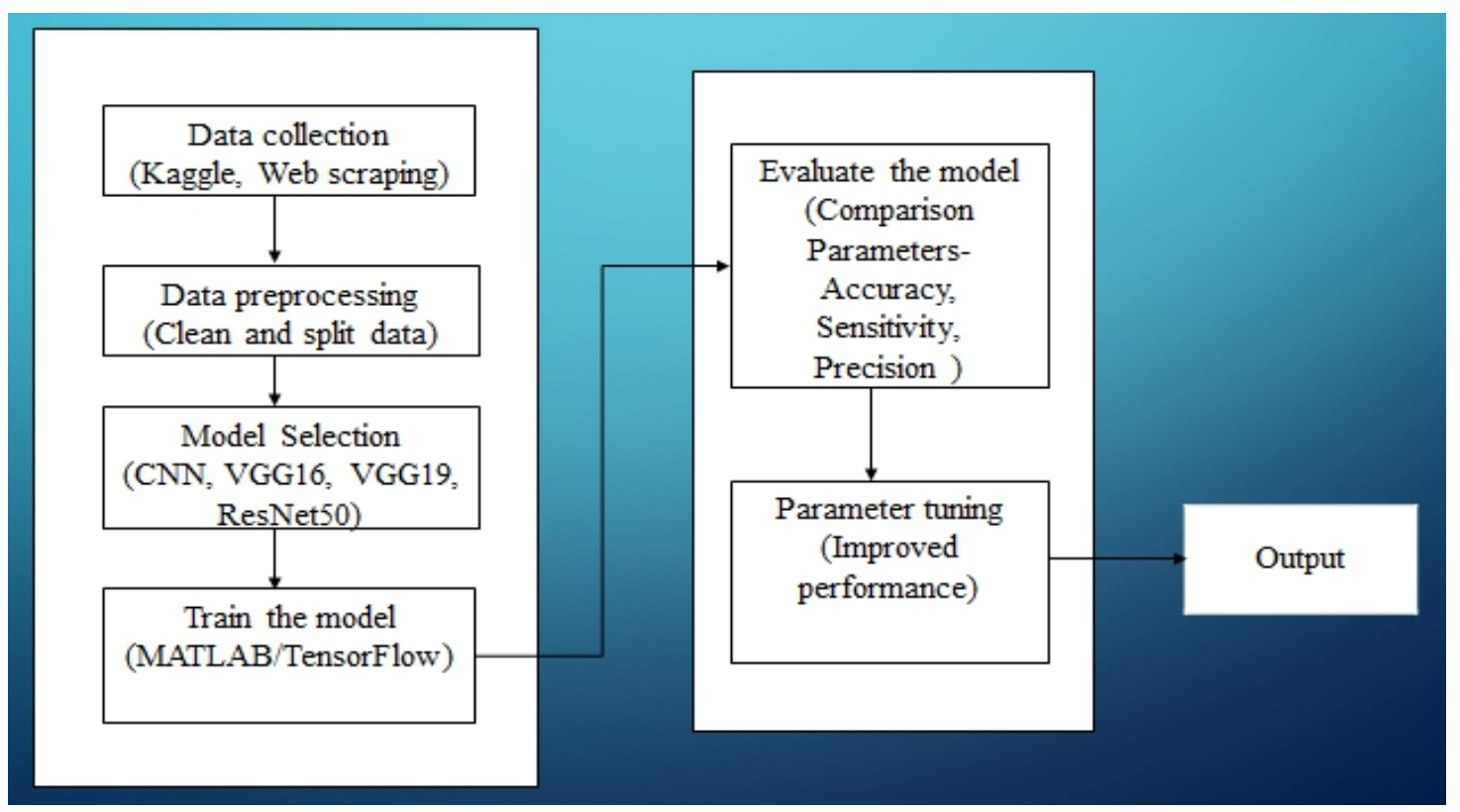

Figure 03: Block Diagram of Proposed System

- Above given is a rough representation of the system that will be followed for the implementation purpose. The architecture is divided into two halves. The first half consists of training and second half consists of testing and actual detection.

- As shown in the first half, a dataset is created using both Kaggle dataset and web scrapping. This created dataset will now be subject to various data cleaning methods where all the null values and garbage data is removed. After the application of chosen deep learning algorithms, a model will be created with set of features. Later this model is used for predicting whether an image consists of brain tumor.

\section{Implementation}

System Implemenation is done starting with data collection. It is followed by other steps as mentioned 
below -

- Data Collection: - Data collection has been done from Kaggle public data set and web scrapping.

- Data Preprocessing: - Images obtained from various sources are collected and kept aside. These images are then scanned, and noise cancellation is performed on them. Also, duplicate images are removed. Also, the extra unwanted images downloaded like human photographs or up or down arrow are discarded. Later, these are converted into black and white images. Black and white image conversion is done by using OpenCV library. After the images are successfully converted into black and white, they are then undergoing the process of data augmentation. In data augmentation, the images are rotated, horizontally flipped, sheared, vertically rotated, zoomed, etc. and then the final dataset of all 5 classes is created. Five classes are being created viz. Class 1Diffusion Tensor Imaging (DTI) scanned brain tumor images, Class 2- Magnetic Resonance Imaging (MRI) scanned brain tumor images, Class 3 - Magnetic Resonance Spectroscopy (MRS) scanned brain tumor images, Class 4 - Normal scanned brain images which includes all of MRI, MRS, PTE and DTI and Class 5 - Positron Emission Tomography (PET) scanned brain tumor images. Also, the data is increased by randomly replicating the data.

- Model Selection: - Once the data is ready for further processing, algorithms are shortlisted on which data will be processed. In this system, all four algorithms are implemented which are CNN, VGG16, VGG19 and ResNet50.

1. VGG19 - VGG19 is a version of the VGG model, which has 19 layers in total (16 convolution layers, 3 Fully connected layers, 5 MaxPool layers and 1 SoftMax layer)

2. CNN - It accepts an image as an input, grants importance to various attributes in the image, and can distinguish between them.

3. ResNet50 - It's abbreviated as The basis of many computer vision tasks is the residual network, which is a classic neural network. ResNet was a game-changer because it helped us to successfully train extraordinarily deep neural networks with 150+ layers.

4. VGG16 - VGG16 is a version of the VGG model that has 16 layers in total (13 convolutional layers, 3 fully connected layers, 5 MaxPool layers and 1 SoftMax layer).

- Training the Model: - Model training is done taking $80 \%$ of the dataset. This is done so that remaining $20 \%$ of the data could be utilized for testing purpose.

- Evaluation of Model: - Model evaluation is done by comparing the parameters obtained as part of result. The parameters used for evaluation are Accuracy, Error Rate, Precision, F1-score and Cohen's Kappa.

- Parameter Tuning: - This is done to improve performance of parameters. 


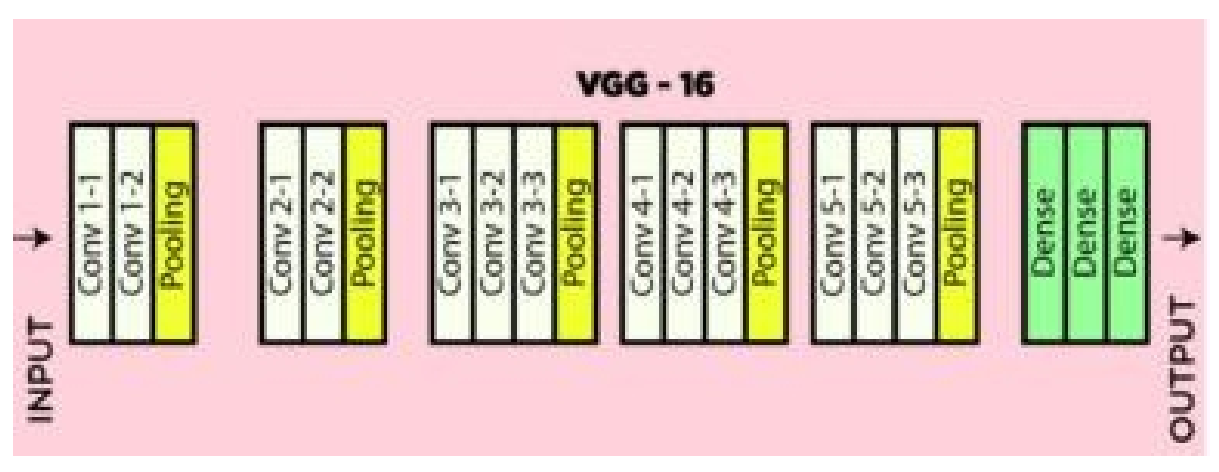

Figure 04: VGG16 Architecture Map

Implemetation of VGG16 has got best results. The network's input is a two-dimensional image (224, $224,3)$. First ever two layers have the same padding and 64 channels of $3 * 3$ filter size. Following a stride $(2,2)$ max pool layer, two layers of 256 filter size and filter size convolution layers are added (3, $3)$. This is accompanied by a stride $(2,2)$ max pooling layer, which is same as the previous layer. Then there are two convolution layers with filter sizes of 3 and 3 and a total of 256 filters. Following that, there are two sets of three convolution layers, as well as a max pool layer. All have 512 filters of the same size $(3,3)$ and padding. This image is then fed into a two-layer convolution stack. The filters we use in these convolution and max pooling layers are $3 * 3$ rather than $11 * 11$ in AlexNet and $7 * 7$ in ZF-Net. It also uses $1 * 1$ pixels in some of the layers to manipulate the set of possible channels. After each convolution layer, 1-pixel padding is applied to prevent the image's spatial attribute from being lost.

The equations performed as part of this algorithm are as follows -

1) The ImageNet dataset comprises RGB-channeled images with a fixed size of $224 * 224$ pixels. As a result, our input is a tensor of $(224,224,3)$. This model takes the input image and generates a 1000 -value vector.

$$
\hat{y}=\left[\begin{array}{c}
\hat{y}_{0} \\
\hat{y}_{1} \\
\hat{y}_{2} \\
\vdots \\
\hat{y}_{999}
\end{array}\right]
$$

Equation 1 - Input Vector

2) The classification probability for the corresponding class is represented by this vector. Assume we have a model that predicts with probability that an image belongs to class 0 . Class 1 has a probability of 0.05 , class 2 has a probability of 0.05 , class 3 has a probability of 0.03 , class 780 has a probability of 0.72 , class 999 has a probability of 0.05 , and all other classes have a probability of 0.05 . So, the classification vector for this will be: 


$$
\hat{y}=\left[\begin{array}{c}
\hat{y}_{0}=0.1 \\
0.05 \\
0.05 \\
0.03 \\
\vdots \\
\hat{y}_{780}=0.72 \\
\vdots \\
\hat{y}_{999}=0.05
\end{array}\right]
$$

Equation 2 - Classification vector

3) We use the softmax function to ensure that these probabilities add up to one. This softmax function's definition is as follows:

$$
\operatorname{Softmax}\left(\varkappa_{i}\right)=\frac{\exp \left(\varkappa_{i}\right)}{\sum j \exp \left(\varkappa_{j}\right)}
$$

Equation 3 - SoftMax Function

4) We then add the five most likely candidates to the vector.

$$
C=\left[\begin{array}{c}
780 \\
0 \\
1 \\
2 \\
999
\end{array}\right]
$$

\section{Equation 4 - Most Probable Canditates}

5) And this is how we describe our ground truth vector:

$$
G=\left[\begin{array}{l}
G_{0} \\
G_{1} \\
G_{2}
\end{array}\right]=\left[\begin{array}{c}
780 \\
2 \\
999
\end{array}\right]
$$

Equation 5 - Ground Truth Vector

6) The Error function is then defined as follows:

$$
\begin{gathered}
E=\frac{1}{n} \sum k \min _{i} d\left(c_{i}, G_{k}\right) \text { Where } \mathrm{d}=\mathrm{oif}_{i}=G_{k} \text { else } d=1 \\
\text { Equation } 6 \text { - Error Function }
\end{gathered}
$$

7) For this case, the loss function is:

$$
\begin{gathered}
E=\frac{1}{3}\left(\min _{i} d\left(c_{i}, G_{1}\right)+\min _{i} d\left(c_{i}, G_{2}\right)+\min _{i} d\left(c_{i}, G_{3}\right)\right) \\
\text { So, } \\
E=\frac{1}{3}(0+0+0) \\
E=0
\end{gathered}
$$

Equation 7 - Loss Function

Since all of the groups in the ground reality are represented in the Predicted top-5 matrix, the loss is zero.

We must deploy on the server in order to achieve the desired result. Following are the classes defined - Class 0 - DTI scanned brain images, Class 1 - Normal brain scanned images, Class 2 - PET scanned 
brain images, Class 4 - MRI scanned brain images.

Home screen will be appearing like this -

\section{Project Deployment Work !!!}

\section{Image Classifier Project}

\section{Choose..}

Figure 05: Home page

- After clicking on the choose button, we need to select image from the dataset. Thereafter, selected image is displayed on the screen with a predict button.

\section{Project Deployment Work !!!}

\section{Image Classifier Project}
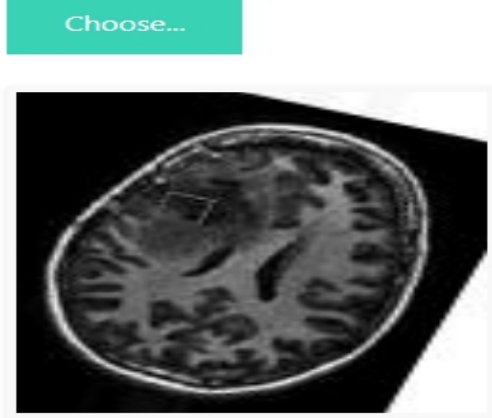

Predict!

Figure 06: User interface with choose and predict button

- Once the predict button is clicked, the system will check to which category the image belong and gives the result. It gives result based on the dataset segregated in 5 classes created. The result displayed is as follows - 


\section{Image Classifier Project}
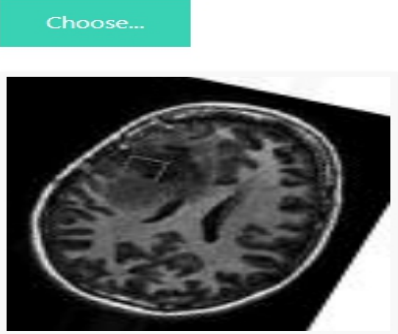

Result: MRS

Figure 07: Result with the classification

\section{Experimental Results}

In order to analyze the results obtained as part of these algorithms, Epochs are executed so that we get to know if the accuracy has improved, and error loss is reduced. Training and Validation accuracy and loss obtained after execution of the best model VGG16 is as below -

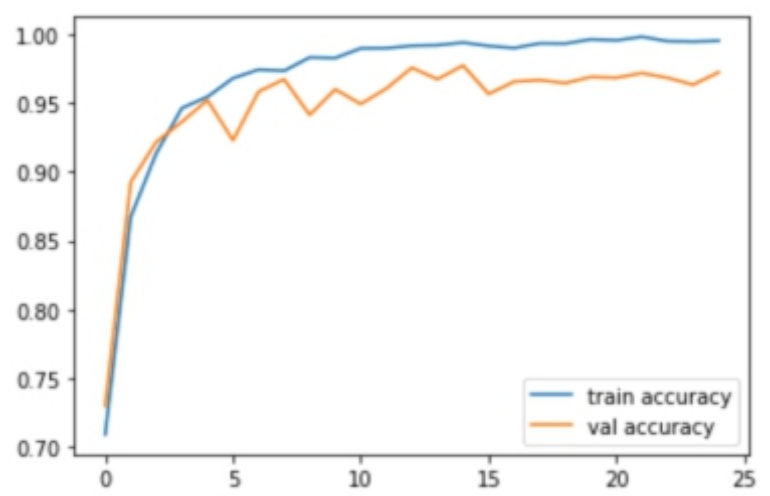

Figure 08: Accuracy obtained of the model

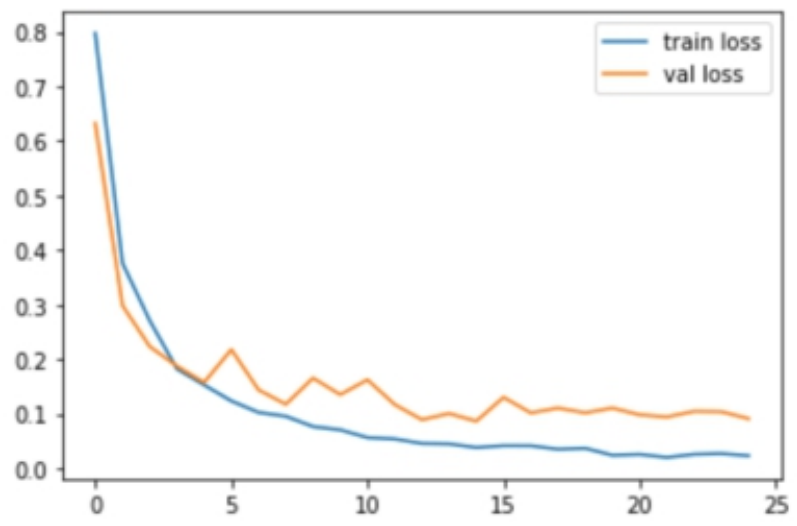

Figure 09: Loss obtained of the model

After this, confusion matrix is applied. It's a table that shows how well a classification model or classifier performs on a collection of test data for which the true values are known. Terminology can be 
International Journal of Intelligent Communication, Computing and Networks

Open Access Journal (ISSN: 2582-7707)

https://doi.org/10.51735/ijiccn/001/23

a bit confusing however; the confusion matrix itself is relatively simple to understand. It's used to represent key predictive analytics including recall, specificity, accuracy, and precision.

Following is the confusion matrix, accuracy, error rate, precision, recall, f1-score, Cohens Kappa obtained in the project as shown as below -

Table 1: Confusion Matrix

\begin{tabular}{|c|c|c|c|c|}
\hline 724 & 32 & 0 & 144 & 6 \\
\hline 32 & 514 & 0 & 59 & 16 \\
\hline 4 & 5 & 154 & 1 & 20 \\
\hline 72 & 23 & 0 & 1489 & 0 \\
\hline 28 & 40 & 5 & 19 & 1075 \\
\hline
\end{tabular}

Accuracy: 0.886598 and Error rate: 0.113402

Classification report:

Table 2: Result of parameters of VGG16 algorithm

\begin{tabular}{|c|c|c|c|c|}
\hline Class & Recall & Precision & F1-score & Support \\
\hline 0 & 0.80 & 0.84 & 0.82 & 906 \\
\hline 1 & 0.83 & 0.84 & 0.83 & 621 \\
\hline 2 & 0.84 & 0.97 & 0.90 & 184 \\
\hline 3 & 0.94 & 0.87 & 0.90 & 1584 \\
\hline 4 & 0.92 & 0.96 & 0.94 & 1167 \\
\hline Accuracy & & & 0.89 & 4462 \\
\hline Macro avg & 0.86 & 0.90 & 0.88 & 4462 \\
\hline Weighted av & 0.89 & 0.89 & 0.89 & 4462 \\
\hline Cohens kappa & 0.846456 & & & \\
\hline
\end{tabular}

Comparison of the parameters of all four algorithms viz., CNN, VGG16, VGG19 and Resnet50 are obtained in the project are as follows as shown in the tables below:

Table 3. Comparison of algorithms for parameters Accuracy, Error rate and Precision

\begin{tabular}{|l|r|r|r|}
\hline Algorithm & Accuracy & Error rate & \multicolumn{2}{|l|}{ Precision } \\
\hline CNN & 0.205737 & 0.794263 & 0.182 \\
\hline VGG16 & 0.889511 & 0.110489 & 0.89 \\
\hline VGG19 & 0.642761 & 0.357239 & 0.636 \\
\hline Resnet50 & 0.319364 & 0.680636 & 0.192 \\
\hline
\end{tabular}

Table 4. Comparison of algorithms for parameters Recall, F1 score and Kappa

\begin{tabular}{|c|c|c|c|}
\hline Algorithm & Recall & F1 & Kappa \\
\hline CNN & 0.202 & 0.072 & 0.002624 \\
\hline VGG16 & 0.894 & 0.892 & 0.851805 \\
\hline VGG19 & 0.61 & 0.598 & 0.530142 \\
\hline
\end{tabular}


Following figure shows the comparison between parameters of all four algorithms -

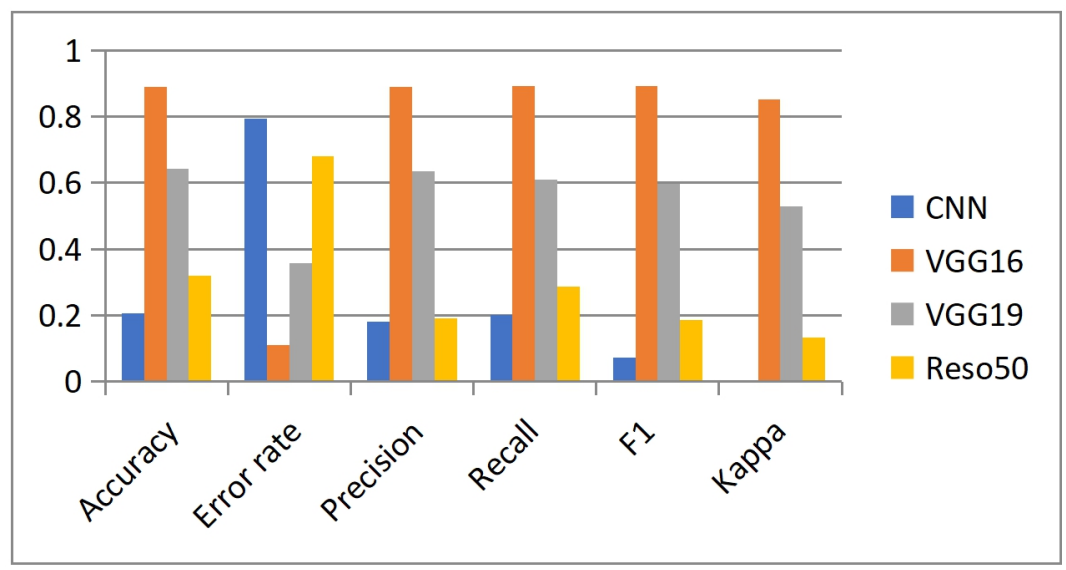

Figure 10: Comparison of the Parameters of algorithms

From the result section it can be seen that the accuracy obtained is highest of the VGG16 algorithm. And the overall detection accuracy of other algorithms when compared is very low. So it can be seen that VGG16 algorithm can successfully boost the over-all accuracy of the system.

The result obtained from VGG16 contained an accuracy of about $\mathbf{8 9 \%}$. The result is quite promising in the healthcare industry.

\section{Discussion}

Using Magnetic Resonance Imaging (MRI), Positron Emission Tomography (PET), Diffusion Tensor Imaging (DTI), and Magnetic Resonance Spectroscopy (MRS) scans, an automated method for segmenting and classifying brain tumours is presented. For a more thorough evaluation, the suggested approach is put to the test at the picture stage. On publicly accessible and web scraping datasets, performance measurements such as Accuracy, Precision, Recall, F1-score, and Cohens Kappa are used to evaluate the suggested process. The proposed technique can be used to improve tumor detection procedures prior to the onset of complications. The results of experiments using all four algorithms, VGG16, VGG19, CNN, and Resnet50, show that the presented approach outperforms previous approaches. This research would aid in the precise and accurate examination of brain tumours.

\section{Acknowledgments}

I would like to take this time to thank my supervisor, Dr. Rajesh Bansode, Professor, TCET, for his keen interest, encouraging direction, and consistent support with my work during the entire process to bring this research to fulfillment.

\section{References}


1. TanzilaSaba, Ahmed Sameh Mohamed, MohammadEl-Affendi, Javeria Amin, Muhammad Sharif, "Brain tumor detection using fusion of hand crafted and deep learning features", 2020 Cognitive Systems Research 59 (2020) 221-230, https://doi.org/10.1016/j.cogsys.2019.09.007.

2. Ambeshwar Kumar, R. Manikandan and Robbi Rahim, 2020 "A Study on Brain Tumor Detection and Segmentation Using Deep Learning Techniques" Journal of Computational and Theoretical Nanoscience, doi:10.1166/jetn.2020.8468.

3. Sidra Sajid, Saddam Hussain \& Amna Sarwar, "Brain Tumor Detection and Segmentation in MR Images Using Deep Learning" 2019, Arabian Journal for Science and Engineering, https://doi.org/10.1007/s13369-019-03967-8.

4. Abd El Kader Isselmou, Guizhi Xu, Shuai Zhang, Sani Saminu, Imran Javaid, "Deep Learning Algorithm for Brain Tumor Detection and Analysis Using MR Brain Images" 2019 Association for Computing Machinery, DOI: https://doi.org/10.1145/3348416.3348421.

5. Heba Mohsen, El-Sayed A. El-Dahshan, El-Sayed M. El-Horbaty, Abdel-Badeeh M. Salem”, 2018 "Classification using deep learning neural networks for brain tumors" Future Computing and Informatics Journal, https://doi.org/10.1016/j.fcij.2017.12.001

6. Javeria Amin, Muhammad Sharif, Mussarat Yasmina, Steven Lawrence Fernandes, "A distinctive approach in brain tumor detection and classification using MRI images" 2017, Elsevier B.V. https://doi.org/10.1016/j.patrec.2017.10.036.

7. AliIşın, CemDirekoğlu, MelikeŞahc, "Review of MRI-based Brain Tumor Image Segmentation Using Deep Learning Methods", 2016 12th International Conference on Application of Fuzzy Systems and Soft Computing, ICAFS 2016, 29-30 August 2016, Vienna, Austria, doi: 10.1016/j.procs.2016.09.407.

8. D.N. Louis , A. Perry, G. Reifenberger, A. von Deimling, D. FigarellaBranger, W.K. Cavenee , H. Ohgaki , O.D. Wiestler, P. Kleihues, D.W. Ellison, The World Health Organization classification of tumors of the central nervous system: a summary, Acta Neuropathol. 131 (6) (2016) 803-820 .

9. Abdel-Maksoud Eman, Elmogy Mohammed, Al-Awadi Rashid, Brain tumor seg- mentation based on a hybrid clustering technique, Egypt. Inf. J. 16 (1) (2015) 71-81, https://doi.org/10.1016/j.eij.2015.01.003

10. Tuhin Utsab Paul, Samir Kumar Bandhyopadhyay, Segmentation of brain tumor from brain MRI images reintroducing K -Means with advanced dual localiztion method, Int. J. Eng. Res. Appl.(IJERA) 2 (3) (2012) 226-231 